\title{
Auditory Reachability: An Affordance Approach to the Perception of Sound Source Distance
}

\author{
Lawrence D. Rosenblum, A. Paige Wuestefeld, and \\ Krista L. Anderson \\ University of California, Riverside
}

\begin{abstract}
The literature on perception of sound source distance reveals a wide range of listener accuracy. Most experiments have listeners perform unintuitive tasks, using unnatural sounds presented in impoverished acoustic environments. The present experiments implement an affordance paradigm for which listeners judge the "reachability" of a natural, live sound source in a familiar acoustic environment. Results reveal that listeners are quite accurate in judging whether the source is reachable and are sensitive to the advantage afforded by two vs. one degree of freedom reaches. Further analyses reveal that when scaled to an intrinsic bodily dimension, judgment differences between listeners disappear, implicating intrinsically scaled specificational information. A follow-up experiment explores the potential informational support for these judgments testing the usefulness of head movements and binaural hearing. Results reveal that whereas head movements had no bearing on either judgment accuracy or consistency, binaural information did enhance listener consistency. This could suggest that the allometric relation between interaural distance and arm length might provide a basis for auditory reachability judgments.
\end{abstract}

Although there is a vast literature on localization of sound sources in the horizontal plane (see Middlebrooks \& Green, 1991, for a review), relatively little research has addressed perception of sound source distance. This is surprising because it is critical for animals to know the location of objects in both planes. For example, a bat uses distance information in timing its interceptive approach to a moth. The same is true of a visually impaired individual guiding his approach to switch off a radio. Sound source distance information is also used in guiding vision. For example, Guski (1992) proposed that knowing the changing distance of a looming object

Requests for reprints should be sent to Lawrence D. Rosenblum, Department of Psychology, University of California, Riverside, CA 92521. E-mail: rosenblu@citrus.ucr.edu. 


\section{ROSENBLUM, WUESTEFELD, ANDERSON}

determines whether there is time to turn and visually localize the object, or whether avoidant behavior must be initiated first. Still, despite the clear ecological importance of the perception of sound source distance, relatively little research has addressed the topic.

The literature that does exist reveals a wide range of performance accuracy. Early research (Edwards, 1955) indicated that thresholds for noticing a change in source distance could be as high as $55 \mathrm{~cm}$ at distances of $1 \mathrm{~m}$, and $1.76 \mathrm{~m}$ for distances of $8 \mathrm{~m}$. Strybel and Perrott (1984) found that at distances of $6 \mathrm{~m}$, listeners needed a change of $36 \mathrm{~cm}$ to hear a difference in distance, whereas at $1.52 \mathrm{~m}$, a change of about $17 \mathrm{~cm}$ was needed. Recently, Ashmead, LeRoy, and Odom (1990) demonstrated impressively low difference thresholds at close distances. They found that at $1 \mathrm{~m}$, practiced listeners had distance change thresholds of $5.8 \mathrm{~cm}$. They attribute this high degree of accuracy to their use of a forced-choice listener task.

It is likely that the wide range of listener accuracy observed across studies is due to considerable differences in experimental design. These differences include the nature of the stimulus tested (e.g., live speech vs. recorded sounds; Gardner, 1968), the nature of the acoustic environment (echoic vs. anechoic; Mershon \& King, 1975), and the nature of the task (method of limits vs. forced-choice; Ashmead, LeRoy, \& Odom, 1990). To help sort out these issues, we report two experiments which attempt to "ecologize" the problem of sound source distance perception. These experiments implement a natural source presented in a familiar acoustic environment, and ask listeners to perform an intuitive judgment task. In addition, we test whether listeners judge distance in relation to their own body scale and explore what the information for body-scaled judgments might be. The theoretical motivation for this paradigm will be presented next, followed by a more detailed description of the methodology.

\section{THE AFFORDANCE PARADIGM}

For Gibson (e.g., 1966, 1979), the objects of perception are not just the objects and events in the world, but also what these things offer or afford an animal. These perceived affordance properties are specific to the animal and its particular set of action capabilities as well as the environmental layout. This perspective drives very different research questions from more standard approaches. For example, the affordance approach examines how animals parse the world perceptually based on anatomical and behavioral constraints. This intrinsic psychophysics contrasts with the extrinsic psychophysics of more traditional approaches which test sensitivities to animal-neutral physical dimensions such as distances, heights, and weights. Instead, the affordance approach asks observers to judge animal referential properties such as whether an object is reachable, traversable, or liftable.

The affordance paradigm has been used successfully to test the visual perception of the traversability of surfaces (Gibson et al., 1987), slopes (Kinsella-Shaw, Shaw, 
\& Turvey, 1992), stairs (Warren, 1984), and apertures (Warren \& Whang, 1987), as well as object reachability (Carello, Grosofsky, Reichel, Solomon, \& Turvey, 1989) and throwability (Bingham, Schmidt, \& Rosenblum, 1990). Affordance research has also been conducted on the haptic perception of reachability (Solomon \& Turvey, 1988; Carello, Fitzpatrick, \& Turvey, 1990), throwability (Bingham, Schmidt, \& Rosenblum, 1990), and the traversability of path gaps (Burton, 1992). This research has revealed that affordances can be perceived to the extent that perceptual judgments are scaled to observers' particular effector dimensions (e.g., leg length for stepability; arm length for reachability). Furthermore, it has been observed that participants are generally more accurate at judging affordance properties than animal-neutral dimensions (e.g., Bingham, Schmidt, \& Rosenblum, 1990).

Integral to this approach is the determination of the body-scaled information that supports affordance perception. Implicit in traditional approaches (e.g., Ullman, 1980, as discussed by Carello et al., 1989) is the assumption that for perceptually guided action, there is a translational step from the extrinsic measurement of an environmental property to the intrinsic metric relevant to the effector organ. In contrast, the affordance approach proffers that environmental information is directly available in an intrinsic form relevant to effector/behavioral properties. This intrinsic information is based on the allometric relations between effector and perceptual system organs. As an example, the lawful relation between eye height and effector dimensions such as arm length and leg length (Davenport, 1944) can support visually informed affordance properties such as reachability (Carello et al., 1989) and stepability (Warren, 1984). It has been shown that information for eye height can be available through either a metric based on where the horizon divides the optic array (Sedgwick, 1983), or based on the optic flow induced by point of observation movement (Lee, 1974). Because of allometric relations, environmental properties can be seen in units of eye height as well as in units of the effector organs. Thus, information about the perceptual system also serves as body-scaled information about the effectors. This allows affordance perception to occur directly without the need for a translational step from an extrinsic to intrinsic informational form.

Although a good deal of research has been conducted on the visual and haptic perception of affordances, very little research has been specifically designed to test auditory affordance perception. (See Gaver, 1993, for work on auditory event recognition from the ecological perspective.) However, it is known that listeners can perceive action relevant properties through sound. This is evidenced by research in obstacle avoidance (e.g., Strelow \& Babryn, 1982; Supra, Cotzin, \& Dallenbach, 1944), aperture traversability (Passini, Dupre, \& Langlois, 1986), and looming sound source avoidance (for reviews, see Guski, 1992; Rosenblum, 1993). The current study uses the affordance paradigm to test accuracy in the perception of sound source distance. In our experiments, we ask listeners to judge whether they can reach a sound source. It was expected that this more intuitive task would 


\section{ROSENBLUM, WUESTEFELD, ANDERSON}

elicit behaviorally appropriate judgments. In this same spirit, the stimulus tested was a natural, live sound presented in a familiar acoustic environment with reflectances. This contrasts with most other auditory distance research ${ }^{1}$ which has used electronically generated stimuli played through loudspeakers and often presented in unnatural acoustic environments (sound attenuated or anechoic chambers).

Some previous research has been conducted on auditory reachability. Clifton and her colleagues (Clifton, Perris, \& Bullinger, 1991; Litovsky \& Clifton, 1992) used a reaching methodology to test sound source distance perception in infants. These researchers tested 7 month olds in a darkened room and used a shaking rattle as the auditory stimulus. They found that infants reached more often for a rattle that was $15 \mathrm{~cm}$ away, than one positioned $60 \mathrm{~cm}$ away. In a follow-up experiment, Litovsky and Clifton (1992) tested adults using a similar methodology. The adults displayed high accuracy in judging these source distances through verbal near-far judgments.

Our experiments borrow from the methodologies of Clifton et al. (1991) and Carello et al. (1989) who examined visual reachability perception. For both experiments, the auditory stimulus is a shaking rattle similar to that used by Clifton et al. (1991). Unlike Clifton's experiments however, we test substantially more than two (near and far) source distances. Also, to examine whether auditory reachability judgments are scaled to arm length, Experiment 1 tests groups of tall and short listeners (Carello et al., 1989). Experiment 1 also applies two different behavioral constraints to the task. Listeners are asked whether they can reach the sound source by (a) simply extending their arm and (b) extending their arm and bending forward at the waist. This manipulation is included to test whether listeners can anticipate the functional consequences of these tasks and perceive the advantage provided by a $2-d f$ reach (Carello et al., 1989).

Experiment 2 was designed to investigate the body-scaled informational basis for auditory reachability judgments.

\section{EXPERIMENT 1}

\section{Method}

Participants. Twenty-four students from the University of California, Riverside participated in the experiment. Most of the participants received credit for an introductory course in psychology; others received payment for their participation. To test the influence of arm length, two participant groups were selected on the basis of their height (Carello et al., 1989). One group consisted of six males

\footnotetext{
'The term auditory distance research (or literature) is used here to refer to the research conducted on the perception of distance through auditory means. The term should not be taken to imply that dimensions of the auditory signal are perceived as such.
} 
and six females of less than $163 \mathrm{~cm}$ in height whereas the other group consisted of six males and six females over $173 \mathrm{~cm}$ in height. Although these two populations exclude a significant portion of the general population, they do provide an efficient means to test the body-scale question. All participants reported good hearing.

Stimuli. The sound source consisted of a rattle made up of a plastic box $(9.75$ $\times 9.75 \times 2.5 \mathrm{~cm})$ containing paper clips. The box was attached to a wooden pole which was inserted into a mechanical chemical shaker. A sound insulating box was constructed around shaker to dampen its sound as much as possible. The shaker rotated the pole approximately $90^{\circ}$ thus rattling the paper clips inside of the box. The rattle oscillated at approximately $5.1 \mathrm{~Hz}$. The average intensity of the rattle shakes was $72 \mathrm{~dB}$ SPL (A-weighted) measured at the listener's head with the rattle positioned $60 \mathrm{~cm}$ away. A spectral display of the rattle sound is shown in Figure 1.

The shaker-rattle apparatus was placed on a metal cart (height $73.5 \mathrm{~cm}$ ) so that the ratcle could be positioned at varying distances from the seated listener. The rattle was positioned in line with the listener's right shoulder at a height of 101.5 $\mathrm{cm}$. The range of distances for the 1 -df condition was from 38 to $110 \mathrm{~cm}$ in $8 \mathrm{~cm}$ increments from the listener's shoulder. The range for the $2-d f$ condition was from 78 to $150 \mathrm{~cm}$ in $8-\mathrm{cm}$ increments from the listener's shoulder.

The experiment was tun in an acoustically normal room $(2.85 \times 2.78 \times 2.74 \mathrm{~m}$ high) which was lightly cluttered. The walls were painted dry-wall, the ceiling consisted of commercial acoustical tile, and the floor was covered with standard linoleum tile. The shaker apparatus moved along the diagonal of this room relative to the listener who was seated in the comer.

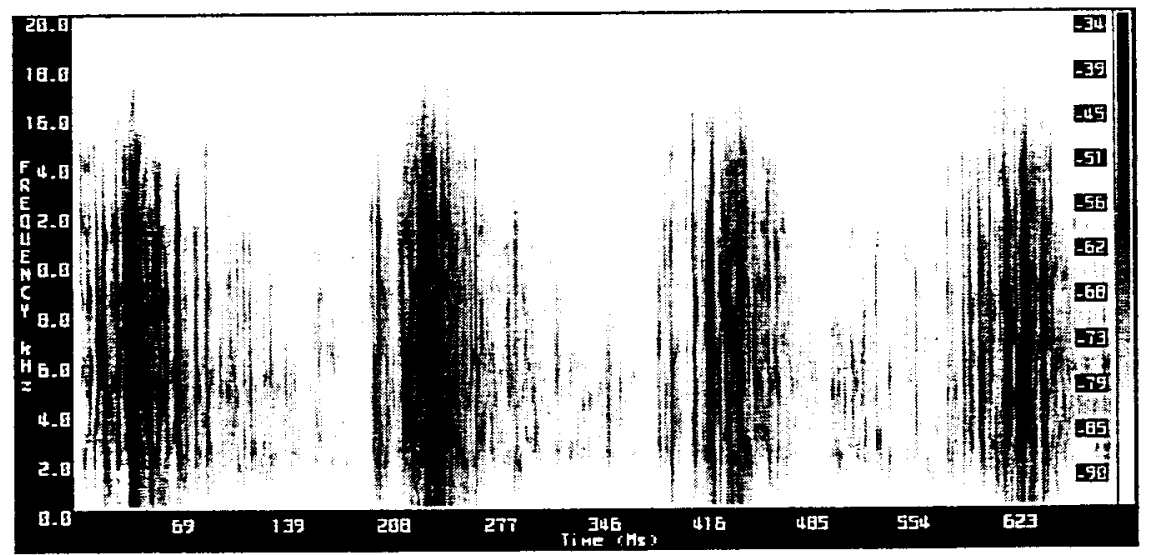

FIGURE 1 Spectral display of four shakes of the stimulus rattle. 


\section{ROSENBLUM, WUESTEFELD, ANDERSON}

\section{Design and Procedure}

When listeners first entered the experiment room, they were told about the experiment and were shown a rattle similar to the one used in the apparatus. Before the trials began, listeners were able to see the room but did not see the actual shaker and cart. Listeners were instructed that for each presentation, they were to judge-without actually reaching-whether the rattle was within the indicated type of reach, 1 or $2 \mathrm{df}$. For the 1 -df condition, listeners were instructed to judge if they could reach the rattle if they were to simply extend their right arm straight out without rolling the shoulder forward and while maintaining contact with the back of the chair. In the 2-df condition, listeners were asked to judge if they could reach the rattle if they were to maximally extend their arm and bend from the hip while maintaining contact with the seat of the chair. These were the same instructions as used in the Carello et al. (1989) experiment. Once listeners provided their reachability judgment, they were asked to rate their confidence in their decision using a 5-point scale ranging from 1 (least confidence) to 5 (greatest confidence). After hearing the instructions, listeners were seated in the chair and blindfolded.

For each trial, the rattle continued shaking until a judgment was made and listeners were given as much time as they needed to make their judgments. Listeners were encouraged to move their head, and told that it might help with their judgment accuracy, but they were not required to perform head movements for the task. Between trials, the listeners heard white noise ( $73 \mathrm{~dB} \mathrm{SPL}$, A-weighted) over headphones to mask the sound of the moving apparatus. Informal pilot studies demonstrated that this noise was effective at masking any information about the direction of the moving cart.

The $d f$ conditions were blocked and half of the listeners received all of the 1-df trials first while the other half received the $2-d f$ trials first. The experiment began with ten practice trials in the initial $d f$ condition. After the practice trials, listeners were given a chance to ask any questions about the procedure. This was followed by three trials each of the ten distances of the $d f$ condition. The trials were randomized with the restriction that no consecutive trials could be at the same distance. The listener was given a short rest halfway through the experiment and told that for the last half of the experiment they were to make their judgments using the other $d f$ condition. For the second half of the experiment, listeners were again given ten practice trials prior to the critical trial set. Listeners received no feedback at any time during the experiment and never touched the apparatus until all judgments were completed. When the experiment was completed, measurements were taken of the listener's arm length (shoulder to tip of thumb) and torso length (shoulder to the seat of the chair). In addition, their maximum reach was determined for each $d f$ condition by having them hold the rattle box out as far as they could while keeping to the constraints of the $d f$ conditions. The experiment lasted about $50 \mathrm{~min}$ for each listener. 


\section{Results and Discussion}

The perceived reach for each listener was calculated by using the furthest distance at which the listener judged the rattle reachable $66 \%$ of the time (two of the three trials; Carello et al., 1989; Heft, 1993). Figure 2 depicts the average perceived and actual reaches for both the short and long armed groups for each of the $d f$ conditions. Figure 3 shows the percentage of responses for which the target was judged reachable for all of the target distances and each of the $d f$ conditions.

Several analyses were performed on the data in an effort to answer two specific questions. First, were listeners making their judgments of source reachability based on their own body scale? Second, were listeners accurate and did their accuracy differ depending on the specific $d f$ condition?

Body-scaled judgments. To assess whether listeners' judgments were based on their own body dimensions, an Order $(1 \mathrm{df}$ first or second) $\times$ Gender (male or female) ${ }^{2} \times$ Arm length (short or long) $\times d f(1$ or 2$) \times$ Reach (Perceived vs. Actual) analysis of variance was performed. Significant effects were found for Arm length, $F(1,20)=12.68, p<01$ and $d f, F(1,20)=991.27, p<01$. Also, a Reach $\times$ Order interaction was significant, $F(1,20)=6.80, p<05$. No other effects or interactions were significant. The significant effect of Arm length indicates that short and long armed listeners had significantly different reaches when averaged over perceived and actual. The significant effect of $d f$ together with the lack of interaction of $d f$ and Reach indicates that listeners' actual reaches were significantly further in the $2 d f$ condition and that listeners perceived this advantage of two degree of freedom reaching. The Reach $\times$ Order interaction indicates that listeners who received the $1 d f$ condition first performed better overall in their reach judgments. It is not clear why this interaction occurred.

To determine whether listeners' judgments were based on their own body scale, we further investigated the effect of Arm length. The significant effect of Arm length shows that on average, long armed listeners judged that they could reach further than short armed listeners (see Figure 2). We reasoned that if listeners' judgments were actually based on their own body dimensions, then when these reach judgments were scaled to an appropriate body measure, judgment differences as a function of arm length differences should disappear. To test this, each listener's perceived reach was divided by arm length in the $1 d f$ condition and arm + torso length in the $2 \mathrm{df}$ condition. The means for these scaled measures were .91 for the short armed group and .94 for the long armed group in the $1 \mathrm{df}$ condition, whereas the means for the $2 \mathrm{df}$ condition were .94 for the short armed group and .91 for the

Gender was tested as a factor in these experiments because we have found gender to play a role in judgment accuracy of other natural auditory events (e.g., Wuestefeld \& Rosenblum, under review). 

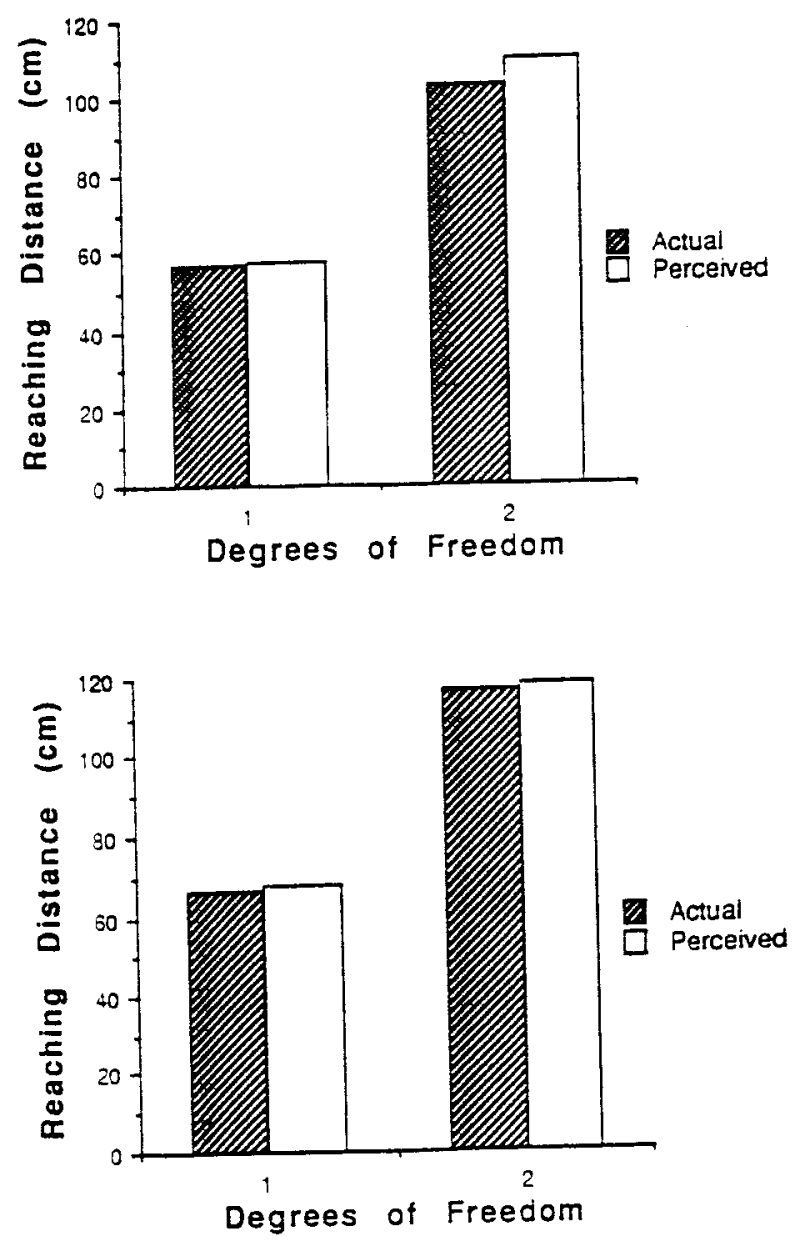

FIGURE 2 Average perceived and actual reaches for short (top) and tall (bottom) listeners.

long armed group. Two t-tests were performed on these scaled measures to contrast short and long armed listeners for each $d f$ condition (Carello et al., 1989). No significant differences were found in either df condition when using these scaled measures, $t(22)=-.4104, p<.05$ for the $1 d f$ condition and $t(16)=.5666, p<.05$ (corrected for unequal variances) for the $2 \mathrm{df}$ condition. These results indicate that short and long-armed listeners perceived reach judgments were similar when scaled to their body dimensions. Thus, as others have found for visually perceived "reachability" (Carello et al., 1989) and stair "climbability" (Warren, 1984) among other examples, our results suggest that there is an intrinsically scaled specificational state which holds over listeners of different sizes. 
Accuracy. Overall listener accuracy was assessed in two ways. First, the effect of Reach was evaluated. The mean perceived-actual Reach difference for the 1df condition was $1.32 \mathrm{~cm}$, whereas this difference for the 2 - $d f$ condition was 3.88 $\mathrm{cm}$. Based on the analysis of variance outlined above, there was no significant effect
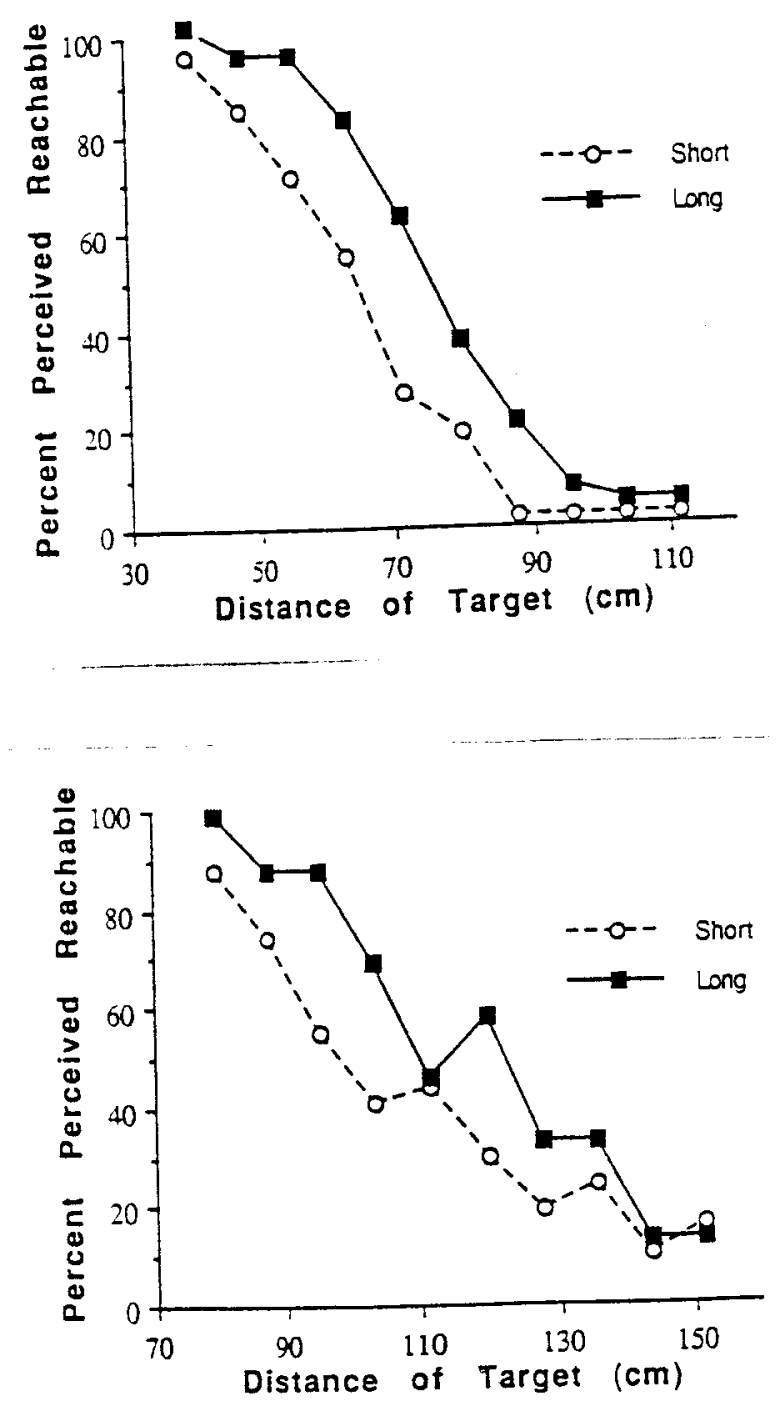

FIGURE 3 Percentage of reachable responses for all target distances for the 1 df condition (top) and the $2 \cdot d f$ condition (bottom). 
of Reach, $F(1,20)=.72, p>.05$. This finding indicates that perceived reach judgments did not differ significantly from actual reach when averaged over listeners. This fact is evident in Figure 2 and suggests that on average, listeners were quite good at judging their actual reach. In addition, the lack of a significant $d f \times$ Reach interaction indicates no difference in accuracy across the different $d f$ conditions. It is interesting to compare our results with those of Carello et al. (1989), which tested visual reaching. Using the same analysis, Carello and her colleagues did find a significant reach effect reflecting that listeners significantly overestimated their perceived reach.

However, the above analysis of variance is potentially misleading as a means of evaluating accuracy. In surveying our data, it became clear that whereas some listeners generally overestimated their reach, many others underestimated. As a result, averaging over listeners may portray judgment results as spuriously more accurate than they are. This was particularly a concern for us in that only $63 \%$ of our listeners overestimated relative to $88 \%$ of the visual reaching observers tested in the Carello et al (1989) study. Based on this concern, an absolute deviation from actual reach was calculated for each listener by determining the absolute difference between perceived and actual reaches. This measure revealed that listeners were off, on average, $10.64 \mathrm{~cm}$ for the $1 \mathrm{df}$ condition and $17.58 \mathrm{~cm}$ for the $2-\mathrm{df}$ condition.

An analysis of variance was performed using this absolute deviation measure as the dependent variable testing the factors of Order ( $1 \mathrm{df}$ first or second), Gender (male or female), Arm length (short or long), and $d f(1$ or 2$)$. This analysis showed significant effects for Arm length, $F(1,20)=4.66, p<.05$, as well as $d f, F(1$, $20)=10.25, p<.01$. No other main effects or interactions were significant. The significant effect of Arm length indicates that the long armed listeners were significantly more accurate overall than the short armed listeners (10.36 vs. 17.87). The effect for $d f$ shows that listeners were more accurate in the 1 - df condition than in the $2-d f$ condition (10.64 vs. 17.58). It is currently unclear why either of these effects occurred. However, it should be noted that overall, listeners seemed somewhat more confident in their $1 d f$ judgments as revealed by mean confidence ratings ( $1 \mathrm{df}=4.0 ; 2 \mathrm{df}=3.3$ ).

The results suggest that reachability judgments were scaled to listener's own body dimensions. The boundary for reaching was the same for both listener groups when scaled to the appropriate effector organs. This implicates intrinsically scaled information which holds over listeners.

With regard to judgment accuracy, our listeners displayed some minor deviations from optimal performance. Still, they did show accuracy comparable to the visual reachability performance reported by Carello et al. (1989). This raises the question: How should accuracy be evaluated in reachability experiments? Carello et al. suggest that judgment accuracy should fall within a tolerance region based on the constraints of the task. For their results, they claim that deviations were within a range such that a performed reach could easily have been fine tuned by small finger adjustments. They state that during an actual reach, there is "on-line" control 
information available. This includes visual information about the relation between the hand and layout, as well as haptic information about the dynamics of the reaching limb. A similar argument has been made by Pufall and Dunbar (1992) who propose that critical points might be better construed as critical ranges-with the choice within the range dependent on exploration. (Relatedly, Konczak, Meeuwsen, and Cress, (1992) show that limb strength and flexibility also plays a role in selecting critical points within a range.)

Carello et ai. (1989) also argue that judgment deviations could be rationalized by relaxing the constraints on the actual reach measure. For their 1-df condition, Carello et al. instructed their observers to reach the target while maintaining contact between the shoulder and the back of the seat. If this constraint had been relaxed and the shoulder were allowed to extend forward, up to $15 \mathrm{~cm}$ extra would be added to the actual reach (Roth, Ayoub, \& Halcomb, 1977). With regard to the current auditory reachability experiment, our listeners were under the same task constraint. It follows then that if this constraint were relaxed, our listeners would also have an extra $15 \mathrm{~cm}$ of potential reach distance. The deviations reported for our results fall well within this tolerance region. Thus, based on these arguments, the auditory reachability performance of the current experiment is functionally accurate.

However, not all researchers maintain that affordance accuracy should be evaluated on a loosely defined functional basis. Heft (1993) has argued that the reaching research (e.g., Carello et al, 1991; Bootsma, Bakker, Snippenberg, \& Tdlohreg, 1992; Heft, 1993) has generally found consistent misjudgments that, on his interpretation, do not fall within a functional tolerance range. He proposes that if body-scaled information exists, listeners should be more accurate on first judg. ments: in some important behavioral contexts, misjudgments could be fatal. Heft argues that one reason observers might not perform well in affordance tasks is that they might be using analytic rather than true perceptual (i.e., direct) judgment strategies. Following the thinking of James (1890) and Dewey (1930), Heft argues that adopting an analytic attitude towards a task inhibits performance. On the other hand, Heft suggests that performing a task in a less conscious, subsidiary way leads to direct perception and optimal performance. Heft presents an experiment providing preliminary evidence that reachability judgments are more accurate when either time limited, or when made a subsidiary part of the rask. Thus, whereas the performance of our listeners would be considered functionally accurate under most interpretations, it would not under Heft's. It should be clear however, that this is not just an issue for auditory reachability, but for affordance research in general.

An additional question arises concerning judgment accuracy: Is the impressive listener performance reported in these results surprising in light of previous auditory distance research? Clearly, it is difficult to directly compare results across experiments, given the novelty of our methodology. However, our methodology is probably closest to those of studies testing absolute distance judgments (e.g., which ask listeners to estimate source distance in feet or inches). When compared to 


\section{ROSENBLUM, WUESTEFELD, ANDERSON}

these studies, our listener accuracy is impressive relative to performance under conditions using anechoic environments (Mershon \& King, 1975; Mershon, Ballenger, Little, McMurty, \& Buchanan, 1989); recorded rather than live sounds (Burler, Levy, \& Neff, 1980); and impoverished stimuli (Molino, 1973).

The current study provides another finding which is not obviously predictable from the auditory distance literature. Our listeners successfully judged distance relative to their own body dimensions. From the perspective of auditory distance research testing extrinsic judgments, this ability would involve a very complicate process. Following the arguments of Carello and her colleagues (1989), an extrinsic metric theory implicates a three step procedure. After computing an absolute distance to a target, an extrinsic-based process must incorporate a second step to compute the reach distance of the effector organ, and a third to compare the quantities derived from these computations. Carello et al. argue that each of these steps involves some representational assumption (e.g., an appropriate body schema) along with some associated error. Further, an extrinsic metric account presupposes that the units for the object and effector organ distances are the same to allow for comparison. The affordance approach on the other hand, involves a single step for perceiving reachability: The observer simply picks up body-scaled, specificational information that is readily available in the structured medium (e.g., sound). Thus, although the auditory distance literature does not explicitly predict results different from those of the current study, the ecological account offers a more parsimonious explanation of our findings.

Experiment 2 was designed to help determine the body-scaled information for auditory reachability.

\section{EXPERIMENT 2}

As mentioned, affordance perception is thought to be based on intrinsic metrics such that there is information that directly specifies environmental properties in terms of body-scaled properties. For example, Carello et al. (1989) suggest that visual reachability can be specified through units of eye height. Analogously, listeners' perception of reachability should also be based on some intrinsic body metric. There are several candidate dimensions to which auditory reachability information might be scaled. The dimensions which hold the most promise are ear height and interaural distance: both are potentially available acoustically and are lawfully related to arm length (Davenport, 1944). Experiment 2 investigates whether reachability is scaled to either of these dimensions.

\section{Acoustic Flow and Ear Height Information}

As stated previously, the optic flow induced by observer movement is informative about eye height (Lee, 1974). Similarly, movement may also inform a listener about his or her ear height: a measure which is anthropomorphically linked to arm length 
throughout growth (Davenport, 1944). Based on the allometric relation between height and arm length, if movement is informative about ear height, then it could also inform about the reachability of sound sources. Thus, if ear height acts as the intrinsic metric for reachability, then listener movements should allow for more accurate reachability judgments.

Past research has tested the influence of listener movement on sound source localization. There is some evidence that for sounds in the horizontal plane, head movements facilitate localization accuracy (Pollak \& Rose, 1967; Thurlow \& Runge, 1967). However, for sound source distance perception, there is conflicting evidence regarding the urility of listener movement. Simpson and Stanton (1973) found no improvement in distance judgment accuracy with head movements. Litovsky and Clifton (1992) found that head movements did not reduce errors in distance judgments when those errors were based on deceptive sound intensities. However, Ashmead, Davis and Northington (1995) and to a lesser degree, Speigle and Loomis (1993) found that movements toward a sound source can facilitate distance judgment accuracy. Thus at this point in time, the utility of listener movement for sound source distance perception is unclear.

What are the types of information available to a moving observer? First, when an observer moves, it is known that time to arrival information becomes available. Visually, the time to arrival of an object approaching an observer (or an observer approaching an object) is specified by the inverse of the relative rate of optical dilation of the object surface (Lee, 1976, 1980). Auditorily, there is evidence that a similar variable based on rate of change information might be available to specify time to arrival (see Lee, 1990; Shaw, McGowan, \& Turvey, 1991; Rosenblum, 1993 for reviews). This variable could be instantiated in a number of acoustic dimensions including the proportional change in intensity, spectral quality and reflectances from a sound source (or reflecting surface) at the point of observation. Importantly, whereas time to arrival is generally construed as a time, it has been proposed that this proportional change can also be construed as a distance (Ashmead et al., 1995). It follows then, that time-to-arrival information could support sound source distance judgments as well. In fact, Ashmead et al (1995) suggest that the moving listeners in their experiment might have used this proportional change information to facilitate greater distance judgment accuracy.

Another type of information available to a moving point of observation is motion parallax. Visually, motion parallax has long been known to provide information for the relative distances of environmental surfaces. In principle, motion parallax information should also be available auditorily (Speigle \& Loomis, 1993). Information specifying the relative distances of sound sources is available in the changing interaural structure (intensity and time) at a moving point of observation (Speigle \& Loomis, 1993). As a listener moves forward, the rate of change of interaural structure generated by a sound emitting or reflecting object (which does not lay in the midsagittal plane) will depend on the distance of the object to the listener. The interaural structure will change more quickly for closer sources than for further ones. 
Although there is evidence that head movements alone might not substantially improve sound source distance judgments (Simpson \& Stanton, 1972; Litovsky \& Clifton, 1993), possibly, when put into the context of our reachability experiment, some improvement will be observed. In Experiment 1, both time-to-arrival and binaural motion parallax information could have been available: the rattle was located outside of the midsagittal plane and listener head movements were not constrained in any way. Thus, it is possible that listener accuracy in Experiment 1 was based on listener movements. This is one of the questions examined in Experiment 2.

\section{Interaural Distance Information}

Another dimension which could act as the intrinsic metric for auditory reachability judgments is interaural distance. Allometric data suggest that there is a direct relation between arm length and cranium size throughout most of development (Snyder, Cook, Karhausen, Nassset, Howells, \& Tipton, 1974). Because cranium size determines the distance between the ears, information about interaural distance could act as scaling information about the reachability of a sound source.

This is reminiscent of analyses of praying mantis and the relationship between interocular distance and perceiving a strikable prey (Michaels, Prindle, \& Turvey, 1985). Citing the work of Maldonado, and others (e.g., Maldonado, Benko, \& Isern, 1970; Maldonado, Rodriquez, \& Balderrama, 1974), Michaels et al. (1985) show how the allometric relation between interocular distance and leg length can provide categorical information to the mantid about when prey are within a strikable distance. In fact, Maldonado et al. (1970) have shown that maximum catching distance is directly related to interocular distance and that two eyes are critical for its determination.

Analogous interaural distance information may be important for judging the reachability of a sound source. If access to interaural distance information is important for reachability judgments, then listeners should perform better when both ears receive sound than when one of the ears is blocked. We have long known that sound source localization in the horizontal plane is substantially more accurate with binaural than monaural hearing (see Middlebrooks \& Green, 1991, for a review). There seems to be less binaural improvement for localization in the vertical plane (Oldfield \& Parker, 1986). Unfortunately, very little is known about the usefulness of binaural structure for distance perception. Coleman (1963) and Hirsch (1968) have both shown that when a sound source is located on either side of the midsagittal plane, ${ }^{3}$ interaural intensity and time differences will be influenced by the source to listener distance, as well as azimuth location (also see Firestone,

\footnotetext{
${ }^{3}$ Although binaural structure for distance is not available when sources are located in the midsagittal plane, this situation is a limiting and unnatural case: Any small head movement displaces the source away from the mid-line rendering binaural structure usable.
} 
1930). In direct evidence that binaural structure might be helpful comes from research of Holt \& Thurlow (1969) as well as Gardner (1969), who found that distance judgments improved as sound source locations deviated from the equatorial plane. However, there is evidence that binaural information does not facilitate accuracy when listeners are asked to judge which of five speakers is emitting a simple tone source (Molino, 1973). It could be, however, that binaural structure will prove useful when tested with a more natural sound and an affordance based task which is directly linked to listener action.

Having shown in Experiment 1 that listeners' judgments of reachability are based on their own body scale, Experiment 2 explores whether the body-scaled information is related to ear-height and/or interaural distance. To this end, the effects of unstructured head movements and binaural information on reachability judgments are investigated. In Experiment 2, listeners were divided into two groups. One group made judgments of reachability based on binaural information, while the other made the same judgments with only monaural information (one ear occluded). Additionally, each group was required to make half of their judgments with head movements, and the other half without head movements. If interaural information is important for reachability judgments, then performance of listeners in the monaural group should be significantly worse than performance of listeners in the binaural group. Additionally, if listener accuracy depends on head movements, then listeners should show some decrement in performance when head movements are inhibited.

\section{Methods}

Participants. Forty students, 24 females and 16 males, participated in Experiment 2 as partial fulfillment of a class requirement. Participants ranged in height from $157.5 \mathrm{~cm}$ to $188 \mathrm{~cm}$. All participants reported having good hearing.

Materials, Design, and Procedure. The same general stimulus and procedure used in Experiment 1 were used in Experiment 2 with some small changes. First, only a one $d f$ reach task was used throughout this experiment. Additionally, the distance range of the rattle was altered. The rattle distances ranged between $40 \mathrm{~cm}$ and $85 \mathrm{~cm}$ in $5 \mathrm{~cm}$ increments from the listener's shoulder.

Participants were randomly divided into two experimental groups. Group 1 participated in the experiment using binaural information to support their judgments. Group 2 made all judgments with only monaural information: Their right ear was occluded. This was accomplished with the use of a commercial foam earplug designed to block $31 \mathrm{~dB}$. In addition, a large, ear-enclosing cotton-filled headphone was placed over the right ear to block further sound. Sound level meter measurements indicated that the earplug together with the headphone attenuated between 35 and $40 \mathrm{~dB}$ (A-weighting). Although this is a substantial reduction in input intensity, this occlusion apparatus could only approximate a true monaural condi- 
tion. In fact, it is more accurate to view this manipulation as attenuating rather than nullifying binaural structure. For convenience however, the occluded ear condition will be referred to as the "monaural condition." Ear condition was implemented as a between subject variable because of the awkwardness of fitting and removing the earplug/headphone apparatus.

To test the effect of listener movement, all listeners (from both groups) participated in two head movement conditions. The Movement condition required listeners to move their head while the rattle was shaking. In this condition, listeners were told that head movements were known to improve judgments, and that they were required to move their head in any way and for however long they wanted during each trial. To limit the movements to only the head and neck, a strap was placed across the listeners' shoulders. In the No Movement condition, listeners were prevented from moving their heads by the use of a bite bar. This bite bar was made up of a small $U$-shaped steel plate which was fastened to a rigid frame. Dental impression compound was attached to the plate and impressions were made for each listener. This ensured a rigid fit between the listeners' teeth and the plate thereby prohibiting head movement. During the head movement condition, the bite bar apparatus was moved forward several inches. The Movement/No Movement conditions were blocked and half of the listeners received all of the movement trials first whereas the other half received the no movement trials first.

The stimulus presentation (rattle shaking) lasted 5 secs after which listeners were required to make a judgment. In both conditions, listeners informed the experimenter about their judgments by giving the "thumbs-up" sign if they judged that they could reach the rattle, and "thumbs-down" if they could not. Listeners then conveyed their confidence ratings by holding up 1.5 fingers based on the scale used in Experiment 1. All other procedural detail in Experiment 2 was the same as in Experiment 1 (e.g., practice trials, 3 trials per condition, no feedback, white-noise mask between trials). When the experiment was completed, measurements were taken of each listener's arm length, from shoulder to tip of thumb; torso length, from shoulder to the seat of the chair; as well as a measurement of their maximum (1 df) reach.

\section{Results and Discussion}

For Experiment 2, the principal questions of interest were whether head movements and/or binaural structure affected listeners' performance in the auditory reaching task. To investigate this issue, both listener accuracy and consistency were evaluated.

Accuracy Data. Once again, perceived reach was calculated for each listener by taking the furthest distance which was judged reachable $66 \%$ of the time. As in Experiment 1 , the absolute difference between perceived and actual reaches was 
calculated for each listener. For listeners who had access to binaural information, the mean deviation value for the No Movement condition was 7.14 whereas the mean for the Movement condition was 8.98. For the listeners who made judgments with one ear occluded, the mean deviation value for the No Movement condition was 8.90 and the mean for the Movement condition was 8.29. These means are comparable to the mean calculated for the $1 \mathrm{df}$ condition of Experiment 1.

To determine whether Ear Condition (monaural vs. binaural) and Movement Condition (movement vs. no movement) affected accuracy, an Order $\times$ Gender Movement $\times$ Ear Condition $(2 \times 2 \times 2 \times 2)$ analysis of variance was conducted using the deviation measure as the dependent variable. In this analysis no significant effects or interactions were found.

Consistency Data. To further explore whether ear condition and/or listener movement influenced reachability performance, a second measure was implemented. It is possible that whereas listeners were equally accurate regardless of whether they had monaural or binaural information or whether they were allowed to move their head, they could be differentially consistent or systematic across the conditions. A listener would be considered highly consistent if he or she made the same judgment over each of the three trials, and highly systematic if he or she judges adjacent distances as reachable a similar proportion of the time. Figure 4 depicts hypothetical data portraying equivalent perceived reach judgments, but differences in systematicity between high (top) and low (bottom). For simplicity's sake, we have chosen to evaluate performance consistency and systematicity as a single variable that will be referred to as "consistency."

To calculate a measure of consistency, each listener's data were transformed to indicate the proportion of "No" responses for each target distance. A probit analysis was then performed to determine the fit of these data to a sigmoid curve. As seen in Figure 4, a highly consistent listener's data will approximate a sigmoid curve, whereas inconsistent data will not fit as well. The probit analysis yields a Chi-square statistic which is used to indicate the probability that the listener's distribution matches the sigmoid curve. The consistency measure tested is simply this Chisquare statistic divided by the $d f s$ involved. Here, high consistency measure values indicate an inconsistent listener (poor fit), whereas low values indicate a consistent listener (good fit). For listeners who had access to binaural information, the mean consistency value for the No Movement condition was .49 and the mean for the Movement condition was .72. For the listeners who made reachability judgments with one ear occluded, the mean consistency for the No Movement condition was 1.25 and the mean for the Movement condition was 1.07 . Recall that a high value indicates inconsistency, whereas a low value indicates consistency.

Tto determine whether Ear Condition (monaural vs. binaural) and Movement Condition (movement vs. no movement) affected consistency, an Order $\times$ Gender $\times$ Movement $\times$ Ear Condition $(2 \times 2 \times 2 \times 2)$ analysis of variance was conducted 

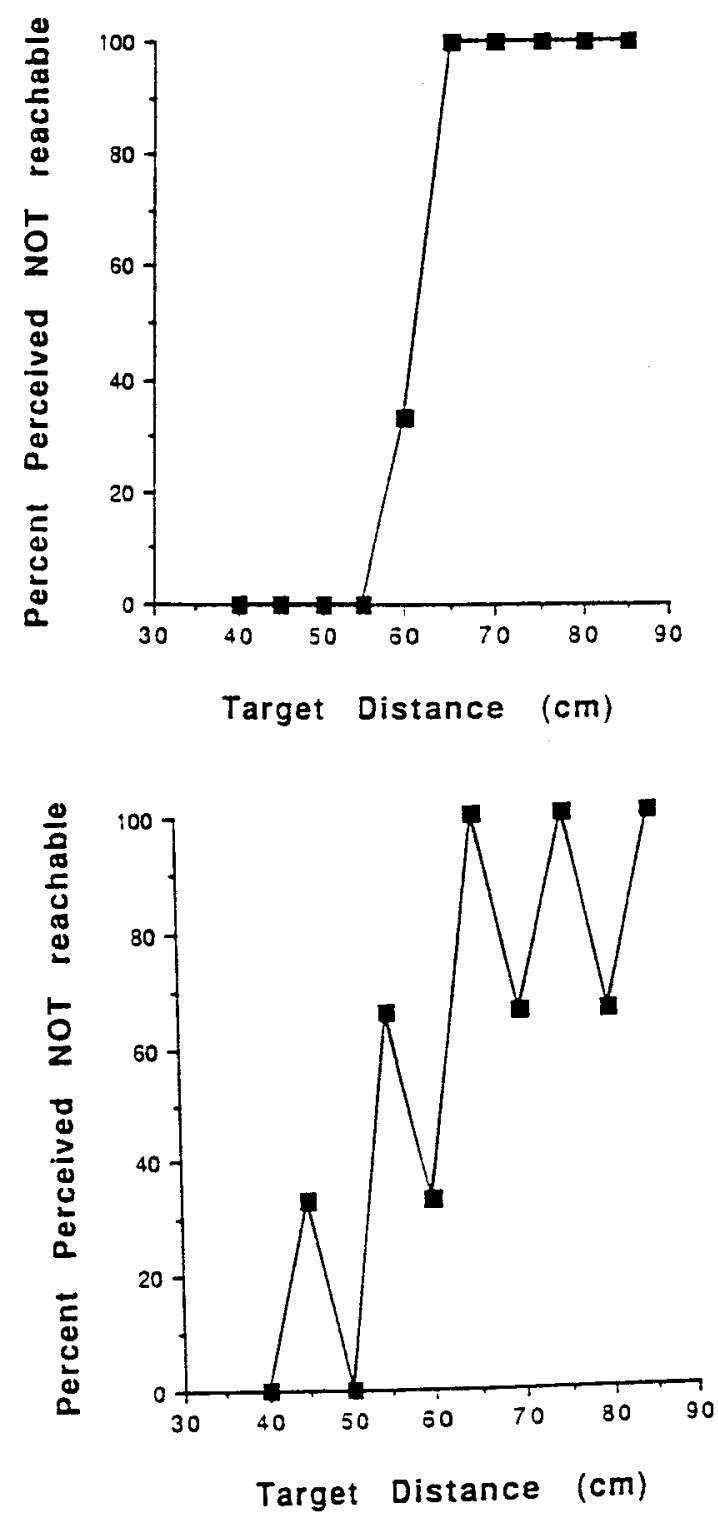

FIGURE 4 Example of high consistency (top) as compared to low consistency (bottom).

using the consistency measure as the dependent variable. This analysis yielded a significant effect for Ear Condition, $F(1,36)=20.14, p<.01$. No other effects or interactions were significant. This effect for Ear Condition indicates that listeners were significantly more consistent with binaural information than with monaural information. This suggests that listeners' performance in this auditory reaching 
task was diminished with the use of only monaural information. The implications of this finding will be discussed below.

In addition to accuracy and consistency measures, listeners' confidence rating were investigated. A $t$-test revealed that listeners were more confident with binaural vs. monaural judgments as reflected in the mean confidence ratings (binaural judgments $=3.59$; monaural judgments $=3.07 ; t(38)=2.54, p<.015$ ).

Overall, these results suggest that head movements did not influence listener judgment accuracy or consistency. These are null results and should be interpreted with caution. Still, these results are in accord with much of the data from auditory distance research (e.g., Simpson and Stanton, 1973; Litovsky and Clifton, 1992). The fact that Ashmead et al. (1995) did find greater accuracy when listeners walked forward suggests that substantial listener movements might be needed before they are useful for distance perception.

On the other hand, the consistency results hint that binaural structure might provide useful information for reachability judgments. Additionally, the fact that there was no interaction of Ear and Movement conditions indicates that head movements did not offset the decrement incurred with monaural listening. Clearly, the monaural influence is subtle. However, the results do invite speculation about interaural distance as the intrinsic metric for perceiving auditory reachability. As mentioned, there is an anthropomorphic relation between arm length and interaural distance (Snyder et al., 1974). Possibly then, auditory space can be measured perceptually in a metric of interaural distance. As mentioned, this fact is analogous to the observation that strikability information is available for the praying mantis by virtue of its interocular distance.

It is unclear why the monaural manipulation would influence consistency and not accuracy. Perhaps our measure of accuracy was not precise enough to detect performance differences. The criteria of 2 of 3 "Yes" responses (Carello et al., 1989; Heft, 1993) might have been too coarse a measure to reveal some performance changes. Another possible explanation for the subtlety of the monaural manipulation lies in our method of ear occlusion. The ear plug and headphone attenuated between 35 and $40 \mathrm{~dB}$ : enough to modify, but not completely mask binaural structure. Because it is likely that the rattle sound remained partially audible through the masked ear, it is possible that the other dimension of interaural structure-interaural time differences-was still available. Potentially then, listener accuracy in the 'monaural' condition remained impressive because interaural time difference information was still available.

\section{GENERAL DISCUSSION}

There are other reasons why disrupting binaural structure might have hindered judgments. For example, binaural structure might serve as an important "egocentric" dimension for distance judgments. According to Mershon and King (1975), 
egocentric acoustic dimensions are those which can support absolute judgments of distance. Egocentric dimensions allow a listener to judge the distance between himself and the sound upon first hearing the source: No other stimulus context is needed. Exocentric dimensions, on the other hand, provide only relative information for distance. Exocentric dimensions are dependent on factors such as source familiarity and do not support accurate initial distance judgments. Instead, accurate judgments based on exocentric dimensions require additional presentations of the same stimulus at different distances.

Of the acoustic dimensions traditionally identified as salient for distance perception (see Coleman, 1963, for a review), sound intensity and spectral structure (experienced as loudness and timbre, respectively) are considered exocentric. These dimensions, as structured at the listener, necessarily depend on both the distance and nature of the source. In fact, there is evidence that familiarity with the source does facilitate accuracy when only these dimensions are provided (e.g., Gardner, 1969). With regard to egocentric dimensions, the relative prominence of reflectances at the listener (experienced as "ambiance" or "distinctness") provides information for distance which does not depend on the nature of the source. In fact, Mershon and his colleagues (Mershon \& King, 1975; Mershon et al., 1989) have shown that source familiarity is not necessary for use of reflectances. With regard to the results at hand, binaural structure also qualifies as an egocentric dimension. Coleman (1963) has argued that binaural structure is independent of the nature of the source. Although not yet tested, it follows that accurate distance judgments should occur with unfamiliar sources as long as binaural structure is available. Perhaps then, binaural structure is a salient dimension for distance perception because it provides absolute information. This could be an additional reason why the monaural manipulation in our Experiment 2 was disruptive for reachability judgments.

There is also evidence hinting that infants can rely on binaural structure for their distance judgments. In a recent demonstration, Litovsky and Clifton (1992) found that 6 -month old infants were not influenced by changes in intensity that compensated for distance and based their reaching attempts on actual source location. The source was located $45^{\circ}$ to the side of the midsagittal plane making binaural structure available. Litovsky and Clifton speculate that the high-frequency components available in their stimulus provided pronounced interaural differences for the infants allowing them to use binaural structure. Because the infants, in essence, performed reaching judgments, binaural structure might have supported bodyscaled information for their affordance task.

The possibility that infants base their distance judgments on egocentric binaural structure is reminiscent of observations made of infant visual distance perception. Bower (1966) reviews a number of experiments which suggest that in judging distance, infants rely less on object-dependent "pictorial" dimensions (e.g., relative size), and more on the robust parallaxes. Unlike pictorial cues, both motion and binocular parallax dimensions are independent of the nature and size of the image: 
the parallaxes are lawfully related to the distance of the object. In this way, the parallaxes are analogous to the egocentric acoustic dimensions of binaural and reflective structure. Perhaps then, infants generally rely most heavily on informational dimensions that have a lawful relation to the environment regardless of (auditory and visual) modality. Furthering the analogy, exocentric dimensions (e.g., intensity) which are dependent on familiarity, are like pictorial cues for sound source distance. And like the pictorial cues, infants might need to learn how to make use of exocentric dimensions through experience.

There is evidence however, that intensity change is powerfully salient for adult listeners (e.g., Ashmead et al., 1990; Coleman, 1963; Mershon \& King, 1975). In the aforementioned Litovsky and Clifton (1992) study, adults were influenced by the compensatory changes in source intensity. It is unclear why adults would find the intensity dimension so salient in so many contexts. It is not obvious how intensity could translate into body-scaled information for affordances. Affordances and their informational support are thought to be relevant to some anatomical dimension of an animal. As reviewed earlier, movement information for ear-height or binaural information for interaural distance could potentially provide bodyscaled information for reachability judgments. These considerations provided the motivation for Experiment 2. Regarding the reported salience of intensity, this acoustic dimension might not prove as salient when put into the context of a body-scaled task. This can easily be tested using the reachability paradigm. Also, if reachability judgments are dependent on body-scaled information such as ear height or interaural distance, then separate manipulations of these anatomical dimensions should have an influence on accuracy (see Marks, 1987, for an analogous manipulation of eye-height). Future research on auditory reachability could be designed to test these questions.

\section{ACKNOWLEDGMENTS}

We gratefully acknowledge the assistance of Steven Reise and Daniel J. Ozer in statistical guidance, as well as the comments of Rainer Guski, William Mace, and two anonymous reviewers.

This research was supported by an intramural grant from the University of California.

\section{REFERENCES}

Ashmead, D.H., Davis, D.L, \& Northington, A. (1995). The contribution of listeners' approaching motion to auditory distance perception. Joumal of Experimental Psychology: Human Perception and Performance, 21, 239-256.

Ashmead, D.H., LeRoy, D., \& Odom, R.D. (1990). Perception of the relative distances of nearby sound sources. Perception and Psychophysics, 47, 326-331. 
Bingham, G.P., Schmidt, R.C., and Rosenblum, L.D. (1990). Hefting for maximum distance thrown: A smart perceptual mechanism. Joumal of Experimental Psychology: Human Perception and Performance, $15,507-528$.

Bootsma, R.J., Bakkar, F.C., Van Snippenberg, F.J., Tdlohreg, C.W. (1992). The effects of anxiery on perceiving the reachability of passing objects. Ecological Psychology, 4, 1-16.

Bower, T.G.R. (1966). The visual world of infants. Scientific American, 215, 80-92.

Burton, G. (1992). Nonvisual judgment of the crossability of path gaps. Joumal of Experimental Psychology: Human Perception and Pefformance, 18, 698-713.

Butler R.A., Levy E.T. and Neff W.D. (1980). Apparent distance of sounds recorded in echoic and anechoic chambers. Joumal of Experimental Psychology: Human Perception and Performance, 6, $745-750$.

Carello, C., Fitzpatrick,, P. \& Turvey, M.T. (1992). Haptic probing: Perceiving the length of a probe and the distance of a surface probed. Percepcion and Psychophysics, 51, 580-598.

Carello, C., Grosofsky, A., Reichel, F., Solomon, H.Y. \& Turvey, M.T. (1989). Visually Perceiving What is Reachable. Ecological Psychology, 1, 27-54.

Clifton, R., Perris, E. \& Bullinger, A. (1991). Infants' Perception of Auditory Space. Developmental Psychology, 27, 187-197.

Coleman, P.D. (1963). An analysis of cues to auditory depth perception in free space. Psychological Bullean, 60, 302-315.

Davenport, C.B. (1944). Postnatal development of the human extremities. Proceedings of the American Philosophical Society, 88, 375-456.

Dewey, J. (1930). Human Nature and Conduct. New York: The Modern Library.

Edwards, A. A. (1955). Accuracy of auditory depth perception in free space. Joumal of General Psychology, 52, 327-329.

Firestone, F.A. (1930). The phase difference and amplitude ratio at the ears due to a source of pure tone. Joumal of the Acoustical Society of America, 2, 260-270.

Gardner, M. B. (1969). Distance estimation of 0 degree or apparent 0 degree -oriented speech signals in anechoic space. Joumal of the Acoustical Society of America, 45, 47-53.

Gaver, W. H. (1993). What in the world do we hear? An ecological approach to auditory event perception. Ecological Psychology, 5, 1-29.

Gibson, E.J., Riccio, G., Schmuckler, M.A., Stoffregen, T.A., Rosenberg, D., \& Taormina, J. (1987). Detection of the traversability of surfaces by crawling and walking infants. Joumal of Experimental Psychology: Human Perception and Performance, 13, 533-544.

Gibson, J. J. (1966). The senses considered as perceptual systems. Boston: Houghton Mifflin.

Gibson, J. J. (1986). The ecological approach to visual perception. Hillsdale, N]: Lawrence Ertbaum Associates. (original work published 1979)

Guski, R. (1990). Auditory localization: effects of reflecting surface. Perception, 19, 819-830.

Guski, R. (1992). Acoustic Tau: An Easy Analogue to Visual Tau? Ecological Psychology, 4, 189-197.

Heft, H. (1993). A methodological note on overestimates of reaching distance: Distinguishing between perceptual and analytical judgments. Ecological Psychology, 5, 255-271.

Helmholtz, H. von (1925). Helmholtz's Treaties on Physiwlogical Optics. (Edited by J. P. Southall). New York: Optical Society of America.

Hirsch, H. R. (1968). Perception of the range of a source of unknown strength. Joumal of the Acoustical Society of America, 43, 373-374.

Holt, R. E. \& Thurlow, W. R. (1969). Subject orientation and judgment of distance of a sound source. Joumal of the Acoustical Society of America, 46, 1584-1585.

James, W. (1890). The Principles of Psychology. New York, Holt.

Kinsella-Shaw, J. M., Shaw, B., \& Turvey, M. T. (1992). Perceiving "walk-on-able" slopes. Ecological Psychology, 4, 223-239. 
Konczak, J., Meeuwsen, H. J., \& Cress, M. E. (1992). Changing affordances in stair climbing: The perception of maximum climbability in young and older adults. Joumal of Experimental Psychology: Human Perception and Performance, 18,691-697.

Lee, D. N. (1974). Visual information during locomotion. In R. B. Macleod \& H. Pick (Eds.), Perception: Essays in honor of J. J. Gibson, (250-267). NY: Cornell University Press.

Lee, D. N. (1976). A theory of visual control of braking based on information about time-to-collision. Perception, 5, 437-459.

Lee, D.N. (1980). Visuo-motor coordination in space-time. In G.E. Stelmach, \& J. Requin (Eds.) Tutorials in motor behavior. New York: North Holland.

Lee D.N. (1990). Getting around with light or sound. In Warren and Wertheim (Eds), Perception and control of self motion. Hillsdaie, New Jersey: Lawrence Erlbaum Associates.

Litovsky, R. Y. \& Clifton, R. K. (1992). Use of sound-pressure level in auditory distance discrimination by 6-month-old infants and adults. Joumal of the Acoustical Society of America, 92, 794-802.

Maldonado, H., Benko, M., \& Isern, M. (1970). Study of the role of binocular vision in mantids to estimate long distances using the deimatic reaction as experimental situation. Zeitschrift für vergleichende Physiologie, 68, 72-83.

Maldonado, H., Rodriquez, E., \& Balderrama, N. (1974). How mantids gain insight into the new maximum catching distance after each ecdysis. Joumal of Insect Physiology, 20, 591-603.

Mershon, D.H. \& King, L.E. (1975). Intensity an reverberation as factors in the auditory perception of egocentric distance. Perception \& Psychophysics, 18, 311-322.

Mershon, D. H., Ballenger, W. L., Little, A. D., McMurty, P .L. \& Buchanan, J. L. (1989). Effects of room reflectance and background noise on perceived auditory distance. Perception, 18, 403-416.

Michaels, C. F., Prindle, S., \& Turvey, M. T. (1985). A note on the natural basis of action categories: The catching distance of mantids. Joumal of Motor Behavior, 17, 255-264.

Middlebrooks, J. C. \& Green, D. M. (1991). Sound localization by human listeners. Annual Review of Psychology, 42, 135-159.

Molino, J. (1973). Perceiving the range of a sound source when the direction is known. Journal of the Acoustical Society of America, 53, 1301-1305.

Oldfield, S. R. \& Parker, S. P. A. (1986). Acuity of sound localization: A topography of auditory space. III. Monaural hearing conditions. Perception, 15, 67-\$1.

Passini, R, Dupre, A., \& Langlois, C. (1986). Spatial mobility of the visually handicapped active person: A descriptive study. Joumal of Visual Impaiment and Blindness, 80, 904-907.

Pollack, I. \& Rose, M. (1967). Effect of head movement on the localization of sounds in the equatorial plane. Perception \& Psychophysics, 2, 591-596.

Pufall, P. B. \& Dunbar, C. (1992). Perceiving whether or not the world affords stepping onto and over: A developmental study. Ecological Psychology, 4, 17-38.

Rosenblum, L. D. (1993). Acoustical information for controlled collisions. In A. Schick (Ed.), Contributions to Psychological Acoustics. Germany: Bibliotheks- und Informationssystem der Carl von Ossietzky Universität Oldenburg.

Roth, J. T., Ayoub, M. M., \& Halcomb, C. G. (1977). Seating console an workspace design: Seated operator reach profiles. Proceedings of the Human Factors Society, 21, 83-87.

Sedgwick, H. A. (1983). Environment-centered representation of spatial layout: Available information from texture and perspective. In E. C. Carterette \& M. P. Friedman (Eds.), Handbook of perception, (pp. 425-458). New York: Academic.

Shaw B. K., McGowan R. S., \& Turvey, M. T. (1991). An Acoustic Variable Specifying Time-to-Contact. Ecological Psychology, 3, 253-261.

Simpson, W. E. \& Stanton, L. D. (1973). Head movement does not facilitate perception of the distance of a sound source. American Joumal of Psychology, 86, 151-160.

Snyder, W. S., Cook, M. J., Nasset, E. S., Karhausen, L. R., Howells, G.P., \& Tipton, I. H. (1974). Repont of the Task Group on Reference Man. Oxford: Pergamon. 


\section{ROSENBLUM, WUESTEFELD, ANDERSON}

Solomon, H. Y. \& Turvey, M. T. (1988). Haptically perceiving the distances reachable with hand-held objects. Joumal of Experimental Psychology: Human Perception and Performance, 14, 404-427.

Speigle, J. M., \& Loomis, J. M. (1993). Auditory distance perception by translating observers. Proceedings of IEEE Symposium on Research Frontiers in Virtual Reality. San Jose, CA, October 25-26.

Strelow, E.D. \& Brabyn, J. A. (1982). Locomotion of the blind controlled by natural sounds. Perception, $11,635-640$.

Strybel, T. Z. \& Perrott, D. R. (1984). Discrimination of relative distance in the auditory modality: The success and failure of the loudness discrimination hypothesis. Joumal of the Acoustical Society of America, 76, 318-320.

Supra, M., Cotzin, M., \& Dallenbach, K.M. (1944). Facial vision: The perception of obstacles by the blind. The American Joumal of Psychology, 57, 133-183.

Thurlow, W. R. \& Runge, P.S. (1967). Effect of induced head movements on localization of direction of sounds. Joumal of the Acoustical Society of America, 42, 480-488.

Ullman, S. (1980). Against direct perception. Behavioral and Brain Sciences, 3, 373-415.

Warren, W.H. (1984). Perceiving affordances: Visual guidance of stair climbing. Joumal of Experimental Psychology: Human Perception and Performance, 13, 371-383.

Warren, W. H. \& Whang, S. (1987). Visual guidance of walking through apertures: Body-scaled information for affordances. Joumal of Experimental Psychology: Human Perception and Performance, 13, 371-383.

Wuestefeld, A. P. \& Rosenblum, L. D. (under review). Perceptual learning in an auditory time to arrival rask. Under review at Perception.

TABLE 1

Spectral Analysis of Stimulus Signal

\begin{tabular}{cc}
\hline Frequency $(\mathrm{Hz})$ & $\mathrm{dB} \mathrm{SPL}$ \\
\hline 63 & 45.8 \\
125 & 45.8 \\
250 & 45.9 \\
500 & 46.2 \\
1,000 & 47.8 \\
2,000 & 54.3 \\
4,000 & 65.7 \\
8,000 & 61.8 \\
16,000 & 47.5 \\
\hline
\end{tabular}

